\title{
Navigating in multi-level buildings: the effect of rotation
}

\author{
Giulia Mastrodonato ${ }^{1 *} \mathbb{D}$, Domenico Camarda ${ }^{1}$, Dino Borri ${ }^{1}$ and Caterina De Lucia ${ }^{1,2}$
}

\begin{abstract}
Background: The existence of the complexity in layout and physical parameters are still under investigation in urban architecture in cognitive science. Current research seems to pay more attention to wayfinding in two-dimensional environments investigating it in public buildings such as hospitals, airports or university departments, where it is more common to experience disorientation. Also, the presence of turning walks or staircases undermines the users' cognitive map. As a consequence, the sense of disorientation would depend on various factors such as the number of ramps, their location and orientation with respect to the building's main façade.

Methods: We carry out two case studies: the first one is a qualitative experiment at the University of Bremen; the second one is a quantitative analysis at the Technical University of Bari. In both studies we select a random sample in which respondents perform a series of tests. . Participants are not familiar with the building in which the experiment is conducted but have some knowledge with the surrounding environment.
\end{abstract}

Results: Main results suggest the importance of building layouts as an important element affecting human cognitive map in wayfinding. They seem to support the hypothesis that the direction of stairs plays an important role on the disorientation when navigating complex three-dimensional environments.

Conclusions: This paper is an attempt to understand how the location of staircases affects disorientation and how this aspect, in turn, affects knowledge acquisition for wayfinding. These findings, coming from experimentations in indoor environments, seem to evoke a peculiar extension to outdoor environments, too. There is fair interest toward convenient investigation for outdoor integration and/or generalization. An intriguing future perspective for the present research would be the extension to the urban level of spatial cognition.

Keywords: Frame of reference, Rotation, Staircases, Wayfinding, ANOVA

\section{Background}

Scientific literature usually looks at spatial environments with different approaches in different domains (Lloyd 2009; Wooldridge and Veloso 1999; Fischer 2000). In urban planning and/or design studies, the interpretation given to the role of space ranges from a passive tank for human actions (anthropization), to a quasi-active agent which represents parts of the human behavior. As in a loop, the latter dependents upon the space dimension (Weyns et al. 2007; Tversky and Hard 2009). In cognition studies, a spatial environment is largely considered from the standpoint of agents' spatial behavior.

\footnotetext{
*Correspondence: julie.mastrodonato@gmail.com

1 Technical University of Bari, Bari, Italy

Full list of author information is available at the end of the article
}

This can be analyzed through low-level (e.g. the search for an object in a given space) or high level (e.g. enjoying spatial aesthetics) cognitive actions (Mockler 1989). Knowledge-based approaches, in urban planning and spatial organization studies, integrate these two cognitive aspects (Schön 1983).

A broad and comprehensive conceptualization of spatial environments, oriented to their representation and management, is an essential stage in building intelligent devices based on ontological layouts. Space organization is an important part of human agents' spatial prerogatives constituted by intricate interplays between sensorial and cognitive attitudes. As a consequence, an investigation on cognitive potentials of human agents' abilities may enhance the understanding of inherent, and often 
cryptic, spatial features. In normal activity processes, human agents are able to conceptualize spaces and use this conceptual framework to design and/or organize spatial processes. Over the last years, in the planning domain, the interest of the international scientific community has focused on urban architectural design and/or layout organization using a series of intriguing and fuzzy cognitive processes (Hofstadter 1995; Arentze and Timmermans 2006).

The way in which users move through this environment is highly dependent on the way they perceive it. As a consequence, designers, architects or planners should take into account several elements when designing an indoor or outdoor environment.

Wayfinding tasks are easier and more efficient when the layout is simple and readable. Duckham and Kulik (2003) show that users do not consider less important the shape and complexity of the path than its length. Weisman (1981) argues that the environment legibility depends on several key features such as the differentiation between different areas, the degree of visual access, the complexity of spatial layout and the signals. Difficulties arise when translational and rotational motions are taken into account to define the spatial environment.

Several times, during the history of architecture, the suggestions and oddities of orientation and rotation perceptions have been deliberatively used by master architects as structural elements of buildings functions. A recent, well-known representative of such approach is Wright's Guggenheim museum and the ramp-based visiting path. Also, a number of examples are present in classical buildings, particularly in Italy, as interesting attempts to integrate misalignments and disorientations in the functionality of monuments or notable buildings. Yet beyond design artifices, the study of translational and rotational motions in everyday agents' navigating behaviors is today considered a critical element of the cognition of spatial environments, for urban planning and design aims.

Current literature recognizes that the pointing ability is affected by physical or imagined translations. However, this seems to worsen with the presence of physical or even imagined rotations (Kelly and McNamara 2008; Presson and Montello 1994; Risier 2002). With the latter, people imagine a different perspective with respect to that from which they learn the environment; this process forces people to align with the new imagined perspective and therefore rotate the initial spatial frame of reference (SFR) (Huttenlocher and Presson 1973; Péruch and Lapin 1993). This process implies a reduction of pointing tasks accuracy and an increase of the time required to align the two SFRs. Translational motions do not involve this cognitive effort.
The present work is rooted to an understanding of a more general research effort, aimed at exploring the conceptualization and representation attitudes of human agents toward space, particularly toward reaching spatial objectives. The main research interest is to investigate two types of agents' cognitive behaviors: (i) Orientation movement (lower-level behaviors) and memories and fantasies (higher-level behaviors) during their interaction with the spatial environment. Furthermore, the present research tests, through qualitative and quantitative approaches, the above investigation with ad-hoc case studies based on a pilot experiment conducted in two building staircase environments.

The remainder of the paper is structured as follows: "An interdisciplinary approach" section illustrates the specific need of an interdisciplinary approach, "Rotation problem in staircases: an experimental approach" section describes the case studies and discusses the main findings; "Conclusions" section summarizes and concludes.

\section{An interdisciplinary approach}

It is widely recognized, in recent literature and in different academic fields, that the comprehension of the context, the space we are moving through, is fundamental to understand human behavior, both individually and socially.

Space could-perhaps should-be designed as a separate entity, with its own dignity, being able to provide a meaning or to produce relationships not necessarily conceived by designers (Wineman et al. 2006).

Cognitive sciences have investigated the processes underlying mental representations of the environment, and the ability to perform operations on these representations. However, Dalton and Hölscher (2006) argue that these sciences, focusing purely on cognitive aspects, have neglected to assess the environment itself and its design. Hence, the need to find a synergy across different disciplines in understanding the relationship between man and environment comes to light.

It is also recognized that wayfinding is an interactive process between users and the surrounding environment with its physical limits: understanding this interaction is essential in order to develop predictive models of human behavior. This interactive process is studied by spatial cognition.

Users moving in the built spaces adopt criteria dictated by specific choices that shape behaviors. These choices stem from a number of factors such as the destination, the purpose of the trip, the planned stops, the route, the layouts' configurational properties, individual preferences, culture, background, familiarity with the environment. Golledge and Gärling (2003) states that each person encodes spatial information in a different manner. 
Conceptual models that people use to successfully complete wayfinding tasks, are different depending on a number of subjective and objective factors. Instead, the conceptual framework gathering all these elements is always the same, i.e., the cognitive map.

The knowledge gained while navigating in unfamiliar environments is stored and integrated in an egocentric mental representation of the environment, although the level of acquisition and the underlying cognitive processes are not yet entirely clear.

Human beings are also able to build up environments' mental representations not directly available to the sight. Quite often they are not based on precise Euclidean coordinates, as either adjacency relations or distances are not respected. The essential support these mental representations provide to navigation allow us, when they are recalled, to know how to reach the destination, or how to move consciously into the environment without resorting to paper maps or drawings. A salient feature is their versatility, i.e. their ability to be adapted when new factors occur, such as impediments, changed conditions in the environment, assessments of the security situation, forcing us to change the planned route (2002).

In the processes of acquisition, encoding and decoding, as well as information storage, spatial cognitive maps undergo a series of distortions that make these mental representations inaccurate or not fully corresponding to reality. They are characterized by fragmentations, distortions, sometimes overlapped elements in different scales, simplifications, addition or omission of details in order to select the information deemed fundamental and to lighten the cognitive load that otherwise people would not be able to endure (Thorndyke and Hayes-Roth 1981; Hochmair and Frank 2000).

Human beings seem to use different navigation strategies and they are able to integrate them successfully using simplified strategies (Tenbrink and Seifert 2011). The approach to more complex tasks requires the evaluation of the available strategies to reduce the number of elements that must be considered simultaneously and lighten the cognitive load.

Peters (2012) suggests that some elements apply better to our mental representations because they are closer to the way we decode and process information. In that work she claims that in mental representations some elements are omitted, simplified or exaggerated to fit the scale or to fit the means of representation used. This incompleteness is critical to reduce the amount of input information; it defines what is called schematization.

In more practical terms of structuring a city cognitive map, Lynch (1960) laid the foundation for researching in spatial cognition. Relying on a careful study of the maps drawn by people and on the investigation of the route descriptions, he could define some basic elements such as landmarks, paths, nodes, edges and districts.

All these aspects should be investigated to understand what kind of processes underlie the construction of $\operatorname{cog}$ nitive maps when rotational motions come into play. In fact, it is also recognized that there are places more difficult to understand and to navigate through, where it is common to experience a sense of disorientation.

The presence of elements involving rotations implies the use of different SFRs; their superimposition requires a cognitive effort which is not needed when simple translations are performed. In the surrounding environment, it is possible to define an allocentric SFR based on prominent local or global landmarks. The objects are positioned relatively to one another creating relations that, although qualitative, improve the environment legibility and navigability even after rotational motions take place. On the other hand, during navigation the interaction between a human body and the environment produce changeable relations deriving from perceptual, visual and proprioceptive flows occurring between the body and the objects.

It is crucial to understand whether the definition of the adopted SFR in spatial representations is limited to the egocentric experience or it maintains some relations with the elements characterizing the environment as its intrinsic axes. Shelton and McNamara (1997) argue that, even with reference to egocentric systems, these systems can assume allocentric connotations because are influenced by prominent directions of space. The lack of privileged directions pushes people to adopt a purely egocentric SFR.

\section{Rotation problem in staircases: an experimental approach}

With reference to the conceptual framework mentioned above, our research focuses on the influence of rotation on orientation, particularly with respect to an element which causes rotations, i.e., staircase. Hölscher et al. (2006), recognize the use of some common strategies. In our study we investigate how the shape of the constructed environment affects spatial knowledge in different places.

We carry out two experiments (Figs. 1, 2). The first one is a pilot (qualitative) study at the University of Bremen. The second one is carried out at the Technical University of Bari and performs a quantitative analysis. In both studies we have investigated the performance of users: (i) When they access stairs directly along their path (case 1); and (ii) After performing a $90^{\circ}$ turn before encountering the staircase (case 2).

The main aim is to assess to what extent the position of the stairs relative to the layout increases disorientation. 

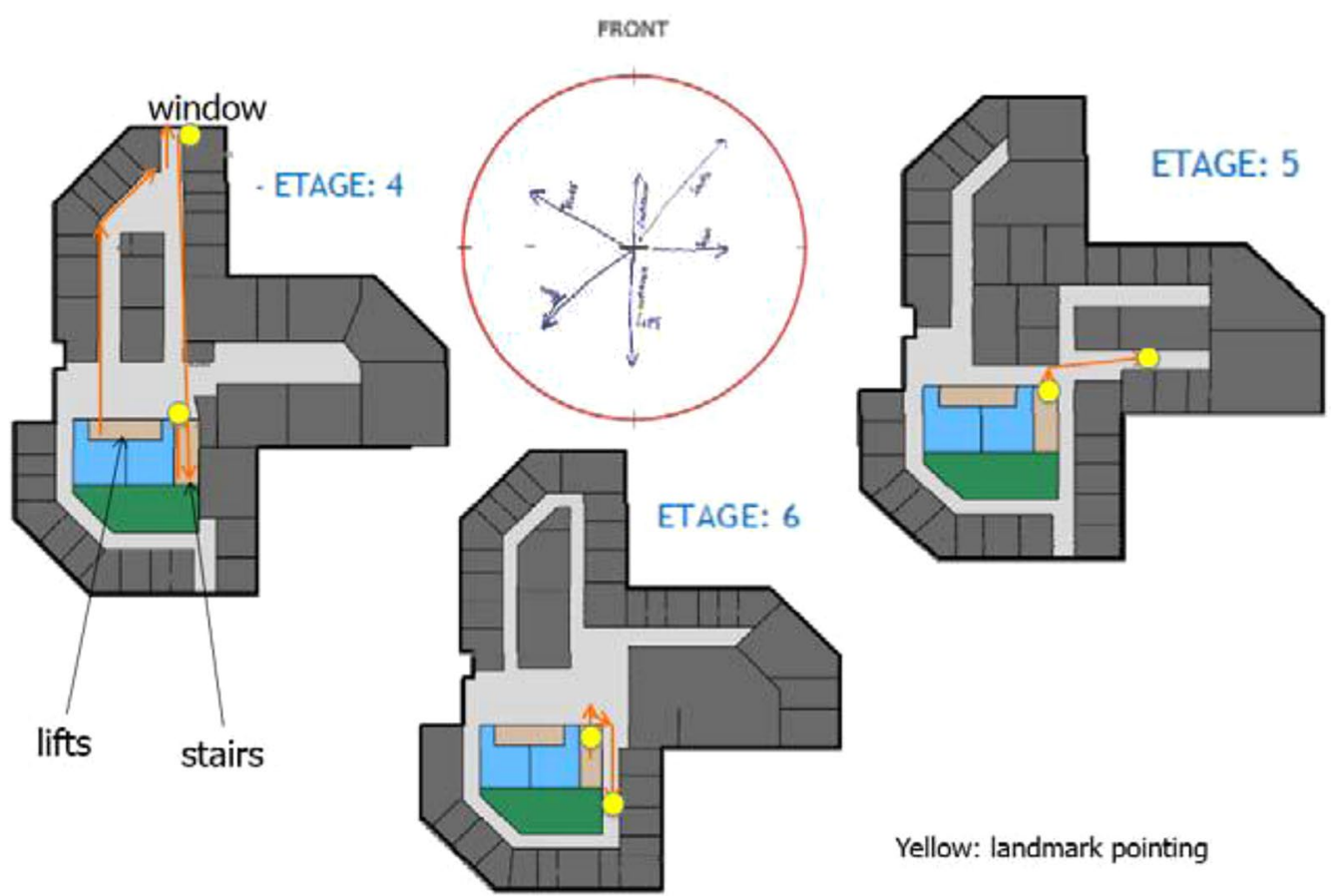

Yellow: landmark pointing

Fig. 1 Floorplans in Bremen: paths for the 1st group

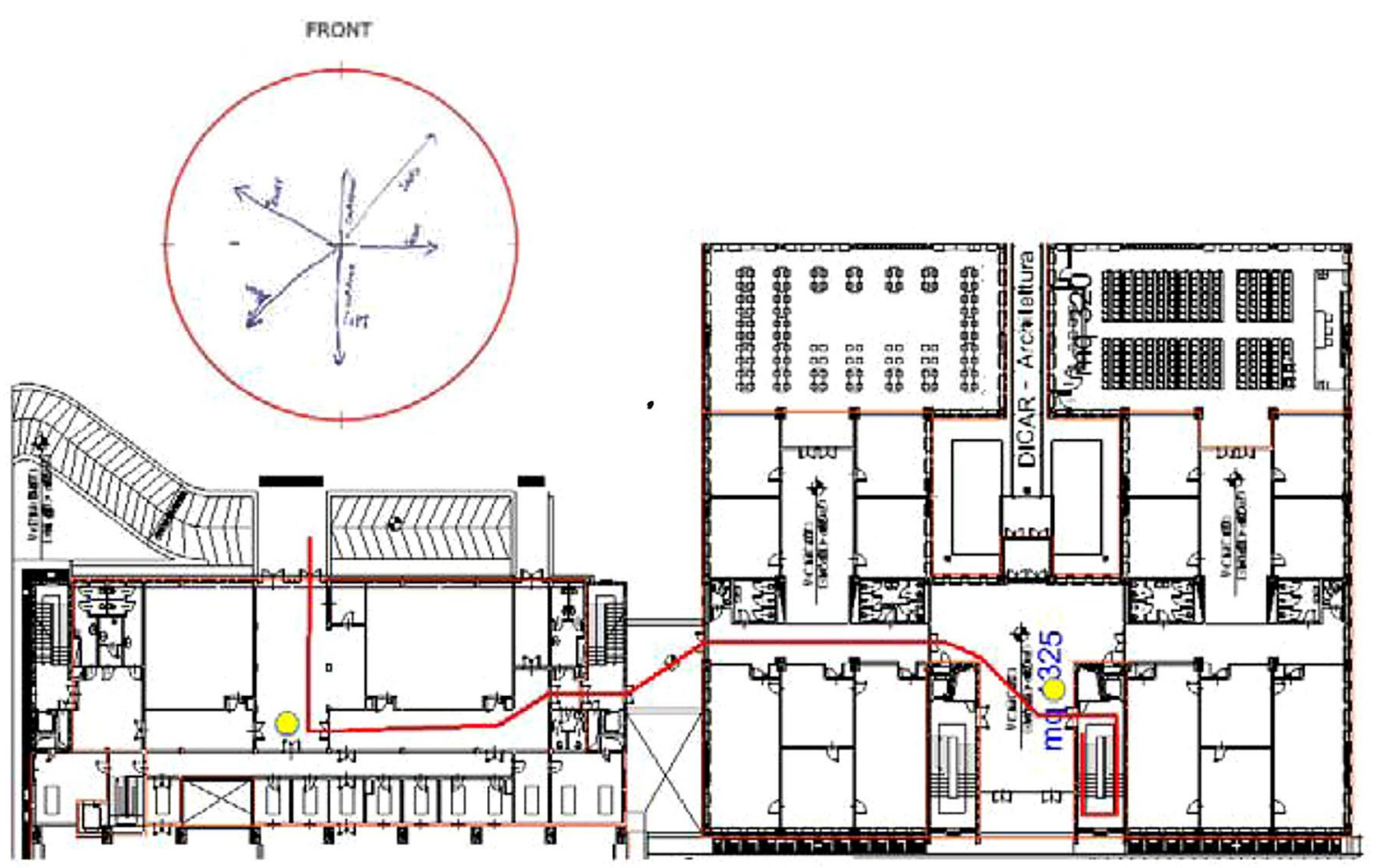

Fig. 2 Floorplan 0 in Bari: paths for the 2nd group

In both universities participants are randomly split into two groups. The first group covers the stairs on the pathway; while the second group turns $90^{\circ}$ before encountering the stairs. To evaluate user's orientation after encountering the stairs we ask respondents to perform some pointing tasks towards local and global landmarks located in 
different points of the building, after a learning phase. In particular, the pointing task is performed by ignoring the vertical dimension of the building. As a consequence, the location of landmarks is spatially aligned at the same level.

According to current literature (Risier 2002), our hypothesis is that the pointing tasks perform better in case 1 than in case 2 . In support of this hypothesis, we argue that when a person is on the top of the stairs, the walkway direction does not change compared to the initial position. Therefore, the egocentric and allocentric SFRs do not conflict.

Furthermore, to assess potential effects due to disorientation when approaching the stairs, we measure the pointing accuracy and the latency. The former is a measure of the configurational knowledge (Richardson et al. 1999); the latter helps to understand the existence of differences between landmarks and the ease of access to the stored information (Montello 1991).

\section{Case study at the University of Bremen}

This section briefly describes the case carried out at the University of Bremen. The sample comprises 30 people randomly chosen. Participants are not familiar with the building in which the experiment is conducted but have some knowledge with the surrounding environment. We split the sample into two random groups. In the first group people approached the stairs on their pathway. In the second group participants need to turn $90^{\circ}$ right to approach the stairs.

Participants, after receiving proper instructions, perform some pointing tasks to analyze the existence of disorientation. The pointing tasks are performed for given landmarks within (local) or nearby (global) the building. Participants are then conducted throughout various levels of the building and are asked to repeat the pointing tasks for each level. It is important to note that each level of the building presents a different layout. The pointing tasks evaluate accuracy and latency of the orientation with respect to the given landmarks. Three pointing tasks are performed.

For the first pointing task, results show a larger mean error when the stairs are covered after turning $90^{\circ}$ than when these are approached directly. As for the latency, the second group seems to take more time to answer to the pointing tasks.

Interestingly, for the second pointing task the mean error is higher in the case when the stairs are directly on the user's pathway than in the case when the users had to perform a $90^{\circ}$ left turn before undertaking the stairs. Our explanation is that having to face a left turn to approach the stairs and then a right turn at the top of the stairwell, participants naturally compensate for the two turns performed on the basis of proprioception information.
The third pointing task sees participants to move to an upper floor. Similarly to the former case we find no relevant differences between groups for both accuracy and latency.

\section{Case study at the Technical University of Bari}

This section shows the case study carried out at the Technical University of Bari. In contrast with the former case, we perform a quantitative analysis with the use of an ANOVA (Analysis of Variance) test. The sample comprises 77 people randomly chosen. The sample is split into two random groups of 39 (group 1) and 36 (group 2) participants, respectively. In this latter group, two participants are left out because do not complete the experiment.

The experiment is similar to that carried out at the University of Bremen and therefore we have that in group 1 people approach the stairs on their pathway; while in group 2, participants turn $90^{\circ}$ right to approach the stairs. Furthermore, participants are not familiar with the building in which the experiment is carried out but have knowledge with the surrounding environment.

Participants conduct the experiment in the same level of the building (contrary to the Bremen case). Also, participants perform pointing tasks for given landmarks within (local) or nearby (global) the building.

In the first pointing task we can argue that there is no statistically significant difference between the two groups in terms of accuracy $(p=0.431)$; for the second pointing task we have a value of $p=0.488$. However, for those who make mistakes the hypothesis is respected. We argue the results as follows: those who face the stairs after turning perceive a rotation on their path but fail to integrate it properly in their cognitive map. Unlike Bremen, the hypothesized compensation of knowledge acquisition does not occur for the Bari case. We believe that this result is due to the difference occurring in the layouts of the two buildings and within each level of the buildings. While in Bremen the layout differs from floor to floor, in Bari it is always the same for each floor. This also implies that the participants in the Bremen case, when moving from level to level, not recognizing the environment of the previous level to be similar to that of the new level, rely only on the elements from their own locomotion.

Contrarily, for the Bari case, the respondents, when exiting from the stairwell, may recognize the environment and rely more on this realization rather than their own memory. As a consequence, they neglect the position of the stairs relative to their path and recognize that they canceled out the rotation movement. Therefore, for the Bari case geometry plays an important role.

The third pointing task, similarly to the other pointing tasks, does not provide statistically significant 
results $(\mathrm{p}=0.473)$ at $95 \%$ C.I in the difference between groups. However, it is worthy to note that the mean error, increases during the first pointing task, decreases during the second pointing task-as if crossing the atrium in some way helps to provide stability to the map-, then increases again during the third pointing task. We argue that this could be simply due to the increased cognitive load. During locomotion, mammals and particularly humans, activate a navigation process that allows the integration of rotations and translations. This provides an estimate of the current position and defines the orientation within a wider environment. Such a process, known as path integration, derives from information received from our senses or proprioception when controlling the movement of the body (Kelly et al. 2008, 2009; Etienne and Jeffery 2004; Philbeck and O'Leary 2005; Kelly and McNamara 2008).

\section{The case for retrieval}

This section illustrates the case for retrieval carried out in the experiments conducted at the Universities of Bremen and Bari.

People are able to update relations to objects even after imagining movements, not only after performing them. This task is in general more complicated and usually requires more time to be assessed. As a consequence, accuracy can also be affected.

Therefore, it is interesting to determine how participants rebuild their cognitive maps. For this purpose, at completion of a navigation tour through the building, participants are taken into a room with no windows. Here are asked to imagine them standing in a precise location of the building and repeat the pointing task from the imagined position.

Once again, we evaluate the accuracy and latency and compare them between the two groups. Literature reports that the pointing tasks from an imagined perspective misaligned with the body are more difficult to perform because the body SFR conflicts with that of the environment (Presson and Montello 1994).

In the case of the first group, this conflict would not occur because participants are asked to imagine themselves, albeit at a different point, in the same perspective from which the environment is learned. In other words, it is as if the mental map is aligned with the environment.

In the second group such alignment would not exist since the position in which participants are asked to imagine themselves is rotated by $90^{\circ}$ with respect to the learning (initial) position.

In both universities, participants in the second group make pointing task mistakes which are almost all approximated to rotations of $90^{\circ}$. This clearly indicates that they do not integrate the position of the stairs rotated by the same angle relative to the position of learning. This seems to confirm the hypothesis.

However, it can also be the case that participants, having acquired some knowledge about the process of the experiment, use specific strategies to retrieve the spatial information and identify landmarks.

As for latencies, these seem to be always slightly higher when stairs require a $90^{\circ}$ turn. This indicates greater difficulty in accessing the information stored. The following figures (Figs. 3, 4) show the case for accuracy (measured in degrees) and latencies (measures in seconds).

Figure 3 shows that the participants of the second group in Bremen have not correctly integrated the direction of the stairs. They, in fact, are rotated $90^{\circ}$ with respect to the direction of the walkway. It is interesting to observe that the accuracy diagrams have approximately the same trend and are phase-shifted by approximately $70^{\circ}$.

Figure 4 shows that almost the same trend is achieved for the retrieval experiment in Bari. This supports the hypothesis that the processes underlying the retrieval of spatial relationships can be considered being similar to those when rotation occurs.

\section{Conclusions}

In general, a crucial problem of urban planning and architecture is to design functional spaces, aesthetically pleasing, easily accessible, taking into account their social impact. For this purpose, it is essential to understand how these environments are used in terms of pedestrian flows, in order to provide decision support systems during the design and the planning phases. As there is a substantial difference in the manner of conceiving spaces from the experts, architects or designers, and lay people, then the understanding of how people live and interact within a constructed environment is the heart of the design choices. Such comprehension can be achieved by a careful observation and monitoring of behaviors.

It is now widely recognized that cognitive maps can be mentally recalled for different purposes during a wayfinding task- such as, remembering a path, estimating a distance or angle, inferring new solutions, planning new ways, making decisions. These operations may imply several operations, among which mental rotation is certainly one of the most delicate.

Montello (1991) suggests that the difficulty in keeping in mind routes with turns, or those ones that are not perfectly straight, can be attributed to the heuristics defined by Tversky (1981). That is to say, spatial memory is influenced by the assumption of reference axes determined by preeminent characteristics of the environment. The author argues that the problem is that the choice of such prominent features, capable of causing observable distortions, is not immediate, and universal, therefore, it 
ACCURACY

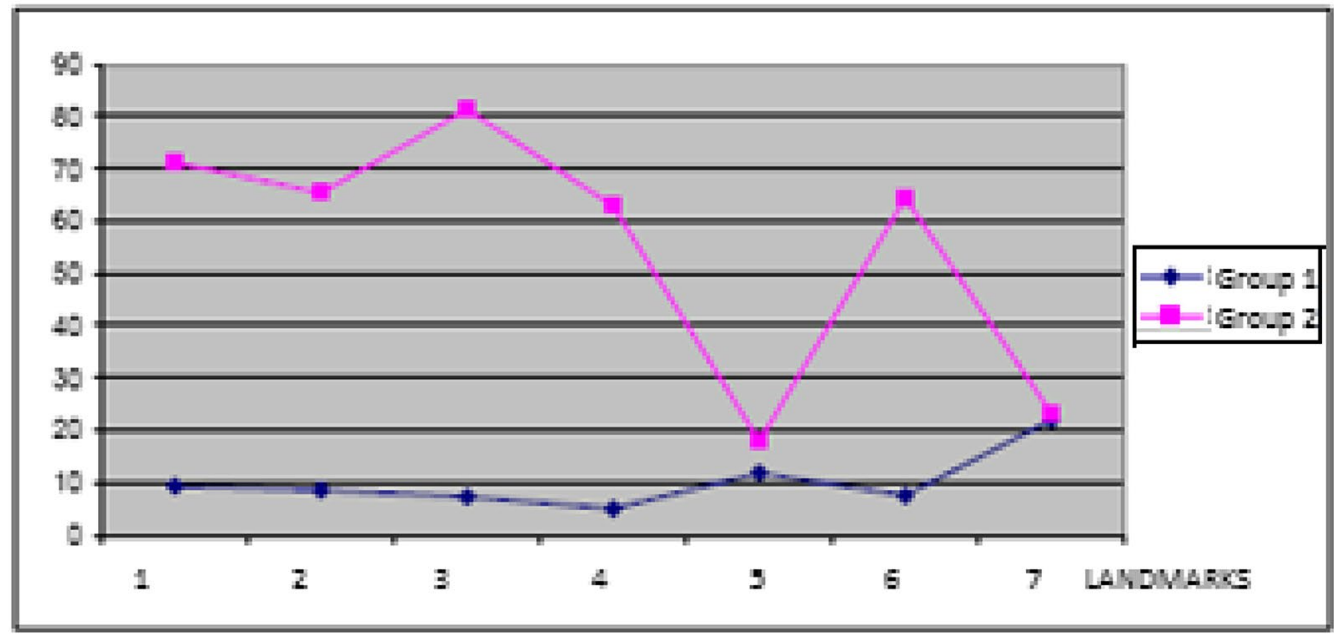

\section{LATENCY}

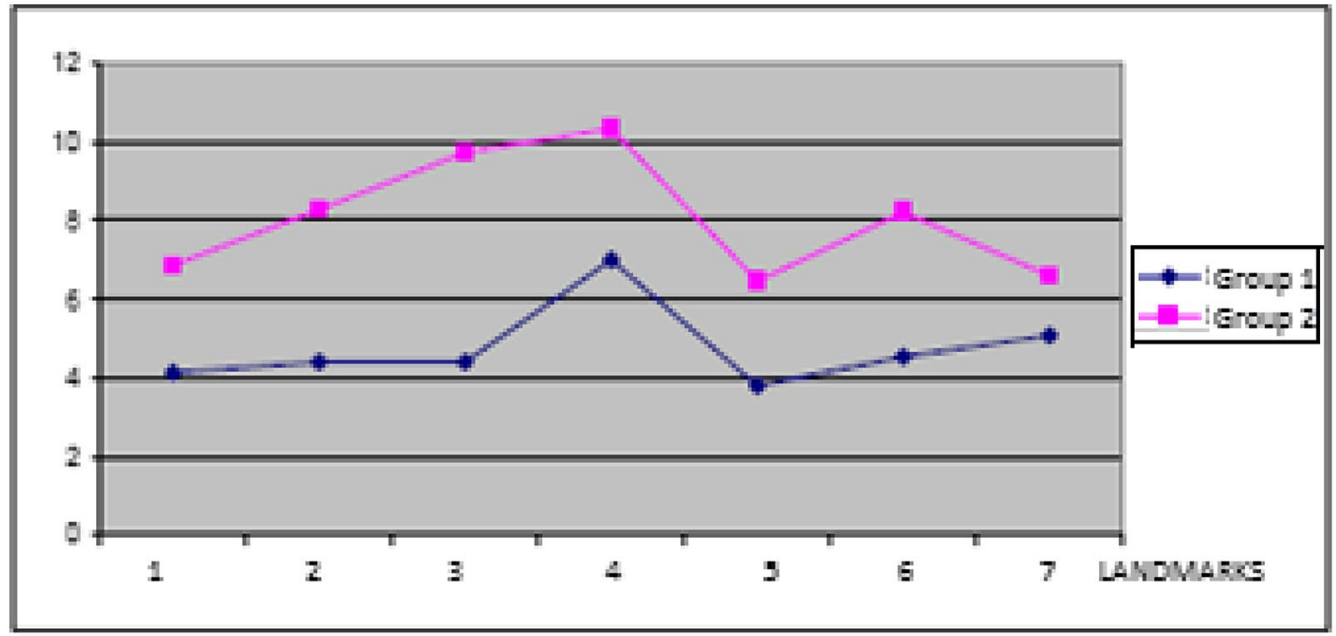

Fig. 3 Comparison between mean errors, in absolute value, during the retrieval for accuracies and latencies in Bremen

does not allow absolute predictions. Moreover, Sadalla and Montello argue that when one performs rotations, it is more difficult to keep the egocentric reference axes aligned with the surrounding environment or with the global references (Sadalla and Montello 1989).

In this framework, the present paper has analyzed the effect of rotation for wayfinding in navigating multilevel buildings. The situations discussed in the present study may actually occur in indoor (staircases, turns) as well as outdoor (e.g. roundabouts) environments: in this case, the experimentation focused on university departments.

We tried to understand whether rotations carried out to cover staircase are perceived as such (i.e. the mental map built up during the navigation is rotated too when spatial relationships are recalled), or not. In the latter case, we tried to understand whether users when identifying their position in the layout of the environment, simply mentally retrace the different segments of the path, regardless of the rotations performed. In both cases, we aimed at understanding how the shape of the built environment affects people's spatial knowledge in different places.

The results seem to support the hypothesis that the direction of stairs plays an important role on the disorientation when navigating complex three-dimensional environments. Results also suggest that people are particularly sensitive to disorientation after rotations due to the presence of stairs. In fact, a preferred environmental direction seems to occur. Furthermore, people 

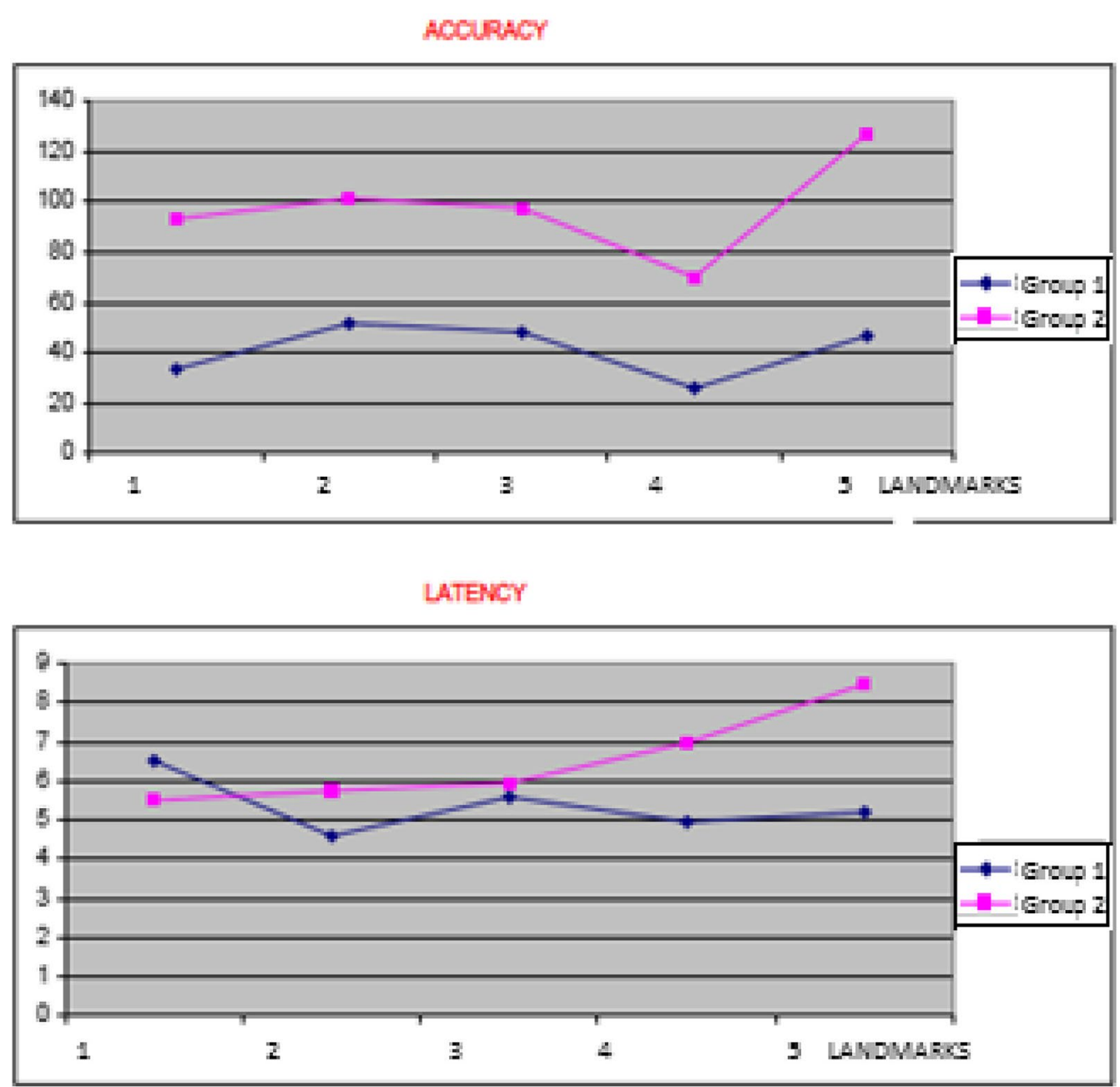

Fig. 4 Comparison between mean errors, in absolute value, during the retrieval for accuracies and latencies in Bari

are unable to get the right orientation between multiple floors after performing turns. We argue that this is due to the following reasons: (i) It is not an easy task for people to integrate, in their cognitive map, different levels during navigation; and (ii) People have difficulties in properly updating their location after performing turns to cover stairs. As a consequence, turns are not perceived as rotations.

This latter aspect should be properly taken into account in emergency situations. Participants probably represent the layout in relation to the reference direction selected during the learning phase and retain this configuration even after the rotations, according to the literature. It suggests that spatial memories may be based on orientation-dependent representations; when instead misalignments occur, conflicts trigger between different SFRs (Tenbrink and Seifert 2011).

The above findings, coming from experimentations in indoor environments, seem to evoke a peculiar extension to outdoor environments, too. In fact, rotation itself is much less relevant to confined spaces (such as buildings) than to open spaces, where possible tracks are rather multidirectional and often unconstrained by narrow pathways and corridors (Borri and Camarda 2009). Turning streets, roundabouts, urban stairways, but also simple multidirectional walking in wider city neighborhoods involve repeated updating of mental maps of directions that can enforce the interest toward the present study approach.

In the end, this circumstance suggests that there is fair interest toward convenient investigation for outdoor integration and/or generalization. This extension to the urban level of spatial cognition represents an intriguing future perspective for the present research.

\section{Author details}

${ }^{1}$ Technical University of Bari, Bari, Italy. ${ }^{2}$ University of Foggia, Foggia, Italy. 
Received: 14 March 2016 Accepted: 19 June 2016

Published online: 08 July 2016

\section{References}

Arentze T, Timmermans H (2006) Multi-agent models of spatial cognition, learning and complex choice behavior in urban environments. In: Portugali J (ed) Complex artificial environments. Springer, Berlin

Borri D, Camarda D (2009) The cooperative conceptualization of urban spaces in ai-assisted environmental planning. Lect Notes Comput Sci 5738:197-207

Dalton RC, Hölscher C (2006) Understanding space: the nascent synthesis of cognition and the syntax of spatial morphologies. In: Hölscher C, Dalton RC, Turner A (eds) Proceedings of the Workshop held in Bremen, Space Syntax and Spatial Cognition, 24 September 2006. Universitat Bremen, Bremen, pp 1-10. ISBN: 978-3-88722-691-7

Duckham M, Kulik L (2003) Simplest paths: automated route selection for navigation. In: Kuhn W,Worboys MF, Timpf S (eds) Spatial information theory: foundations of geographic information science. Number 2825 in Lecture notes in Computer Sciences. Springer, Berlin, p 182-199

Etienne AS, Jeffery KJ (2004) Path integration in mammals. Hippocampus 14:180-192

Fischer F (2000) Citizens, experts, and the environment: the politics of local knowledge. Duke University Press, Durham

Golledge RG, Gärling T (2003) Spatial behavior in transportation modeling and planning. In: Goulias K (ed) Transportation system planning: methods and applications, Chapter III. CRC Press, New York, pp 1-22

Hochmair H, Frank AU (2000) Influence of estimation errors on wayfindingdecisions in unknown street networks - analyzing the least-angle strategy. Spat Cognit Comput 2:283-313

Hofstadter DR (1995) Fluid concepts and creative analogies. Basic Books, New York

Hölscher C, Brösamle M, Vrachliotis G (2006) Challenges in multi-level wayfinding: a case-study with space syntax techniques. In: Hölscher C, Dalton RC, Turner A (eds) Proceedings of the Workshop held in Bremen, Space Syntax and Spatial Cognition, 24 September 2006, pp 143-161. Universitat Bremen, Bremen. ISBN: 978-3-88722-691-7

Huttenlocher J, Presson JC (1973) Mental Rotation and the Perspective Problem. Cogn Psychol 4:277-299

Kelly JW, McNamara TP (2008) Spatial memory and spatial orientation. In: Freksa et al. (ed): SC 2008, LNAI 5248, pp 22-38, Springer, Berlin

Kelly JW, McNamara TP, Bodenheimer B, Carr TH, Rieser JJ (2008) The shape of human navigation: how environmental geometry is used in maintenance of spatial orientation. Cognition 109:281-286

Kelly JW, McNamara TP, Bodenheimer B (2009) Individual differences in using geometric and featural cues to maintain spatial orientation: cue quantity and cue ambiguity are more important than cue type. Psychon Bull Rev 16(1):176-181
Lloyd R (2009) Spatial cognition: geographic environments. Springer, Amsterdam

Lynch K (1960) The image of the City. MIT Press, Cambridge, MA

Mockler RJ (1989) Knowledge-based systems for management decisions. Prentice-Hall, Upper Saddle River

Montello DR (1991a) Spatial orientation and the angualarity of urban routes. A field study. Environ Behav 23:47-69

Montello DR (1991b) Spatial orientation and the angualarity of urban routes. A field study. Environ Behav 23(1):47-69

Péruch P, Lapin EA (1993) Route knowledge in different spatial frames of reference. Acta Psychologica 84:253-269

Peters D (2012) Cognitive principles of schematisation for wayfinding assistance. Ph.D. thesis. Universität Bremen

Philbeck JW, O'Leary S (2005) Remembered Lndmarks Enhance the Precision of Path integration. Psicológica 26:7-24

Presson CC, Montello DR (1994) Updating after rotational and translational body movements: coordinate structure of perspective space. Perception 23:1447-1555

Richardson AE, Montello DR, Hegarty M (1999) Spatial knowledge acquisition from maps and from navigation in real and virtual environments. Mem Cognit 27(4):741-750

Risier JJ (1989) Access to knowledge of spatial structure at novel point of observation. J Exp Psychol Learn Mem Cogn 15(6):1157-1165

Sadalla and Montello (1989) Remembering Changes In Direction. Environ Behav 21(3):346-363

Schön DA (1983) The reflexive practitioner. Basic Books, New York

Shelton A. L., McNamara T.P.: Multiple Views of Spatial Memory. Psychonomic Bulletin \& Review 1997, 4 (1), 102-106 (1997)

Tenbrink T, Seifert I (2011) Conceptual layers and strategies in tour planning. Cogn Process 12(1):109-125

Thorndyke P, Hayes-Roth B (1981) Differences in spatial knowledge acquired from maps and navigation. Cognit Psychol 14:560-589

Tversky B (1981) Distortions in memory for maps. Cogn Psychol 13:407-433

Tversky B, Hard BM (2009) Embodied and disembodied cognition: spatial perspective-taking. Cognition 110:124-129

Weisman J (1981) Evaluating architectural legibility: way-finding in the built environment. Environ Behav 13(2):189-204

Weyns D, Omicini A, Odell J (2007) Environment as a first class abstraction in multiagent systems. Auton Agent Multi Agent Syst 14:5-30

Wineman J, Peponis J, Conroy Dalton R (2006) Exploring, engaging, understanding in museums. In: Hölscher C, Dalton RC, Turner A (eds) Proceedings of the workshop held in Bremen, space syntax and spatial cognition, 24 September 2006. Universitat Bremen, Bremen, pp 33-51. ISBN: 978-3-88722-691-7

Wooldridge M, Veloso M (eds) (1999) Artificial intelligence today: recent trends and developments. Springer, Heidelberg

\section{Submit your manuscript to a SpringerOpen ${ }^{\odot}$ journal and benefit from:}

- Convenient online submission

- Rigorous peer review

Immediate publication on acceptance

- Open access: articles freely available online

- High visibility within the field

- Retaining the copyright to your article

Submit your next manuscript at springeropen.com 